$\mathrm{E}$ EVALUAR
2019, Vol. 19, No. 1

ISSN 1667-4545

Recuperado de https://revistas.unc.edu.ar/index.php/revaluar

Laboratorio de Evaluación Psicológica y Educativa Facultad de Psicología - Universidad Nacional de Córdoba

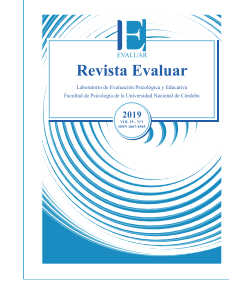

\title{
Validación de la Escala de Esperanza Disposicional para Adultos en Paraguay
}

\section{Validation of the Dispositional Adult Hope Scale in Paraguay}

\author{
M. Alexandra Vuyk * 1 , Gerónimo Codas ${ }^{1}$ \\ 1 - Departamento de Psicología, Facultad de Filosofia y Ciencias Humanas, \\ Universidad Católica Nuestra Señora de la Asunción, Asunción, Paraguay.
}

\author{
Introducción \\ Método \\ Resultados \\ Discusión \\ Conclusión \\ Referencias
}

Recibido: 24/09/2018 Revisado: 14/01/2019 Aceptado: 10/02/2019

\section{Resumen}

Hace algunas décadas, la esperanza disposicional se propuso como un constructo bifactorial. Sin embargo, evidencia reciente sugiere que podría entenderse mejor con un modelo unifactorial. En el presente estudio, mediante análisis factoriales confirmatorios se probó el ajuste de estructuras unifactoriales, con dos factores correlacionados, con dos factores más un factor de segundo orden, y bifactor, de la Escala de Esperanza Disposicional para Adultos (Snyder et al., 1991), traducida al español y adaptada a la población paraguaya. Se realizó el cuestionario a 219 estudiantes universitarios de Paraguay (edad promedio de 23 años; $\mathrm{DE}=3.717 ; 55$ participantes de sexo masculino y $163 \mathrm{de}$ sexo femenino). Los resultados indican un excelente ajuste para los tres modelos, considerándose preferible el modelo bifactor debido a que los índices complementarios indican que la escala es primariamente unidimensional. La escala demuestra propiedades psicométricas adecuadas para su uso en poblaciones latinoamericanas.

Palabras clave: Análisis factorial confirmatorio, modelos bifactor, corrección Satorra-Bentler, Escala de Esperanza Disposicional para Adultos, adaptación transcultural

\begin{abstract}
Trait Hope has been proposed decades ago as a bifactorial construct. Nevertheless, recent evidence has supported the idea of a unifactorial model. In this study, we present results for confirmatory factor analyses in which we assess fit for models that include unifactorial, two correlated factors, two factors with a higher order factor, and bifactor structures of the Adult Trait Hope Scale's (Snyder et al., 1991) adaptation to a Spanish-speaking Paraguayan population. The sample is constituted by 219 college students in Paraguay (mean age 23 years old; $S D=3.717 ; 55$ of the students are male and 163 are female). Results show excellent fit for all three models, with a preference for the bifactor model as complementary indices show the scale is primarily unidimensional. The scale shows adequate psychometric properties for its use in Latin American populations.
\end{abstract}

Keywords: Confirmatory factor analysis, bifactor models, Satorra-Bentler correction, Adult Trait Hope Scale, cross-cultural adaptation 


\section{Introducción}

Hace casi tres décadas, Snyder et al. (1991) plantearon el concepto de esperanza como un dispositivo cognitivo basado en dos conceptos recíprocamente determinados: agencia y medios. Ambos conceptos constituyen la base del modelo propuesto por dichos autores: la agencia, por un lado, puede entenderse como la determinación o motivación hacia una meta; los medios, por otro, son la serie de recursos o estrategias a través de las cuales el individuo pretende la obtención de dicha meta. Además, los autores no conceptualizan la esperanza como un fenómeno que se da distintamente dependiendo de cada meta y contexto en particular, sino como un rasgo o disposición que define la actitud con que los individuos asumen sus objetivos.

De acuerdo con la idea de Snyder (2002), la esperanza es una forma de pensar que se aprende en la infancia a través de la consecución y no consecución de metas. Los individuos con altos y bajos indicadores de esperanza asumen las tareas de modos cualitativamente distintos, y dan distintas respuestas emocionales ante los resultados. Las emociones que aparecen durante el proceso de fijar una determinada meta y las posibles estrategias para aproximarse a la misma retroalimentan al dispositivo de la esperanza, determinando las vivencias que se tendrá en experiencias futuras.

Relacionando la esperanza con otros constructos, Snyder, Feldman, Taylor, Schroeder y Adams (2000) propusieron que la esperanza amplificaría la satisfacción en los individuos. En esta línea, se ha presentado evidencia (Yarcheski \& Mahon, 2014) de la relación positiva entre la esperanza y la satisfacción con la vida. Recientemente, el concepto de esperanza ganó relevancia en el estudio de la orientación vocacional, donde se toma como un factor fundamental en las conductas de exploración (Hirschi, Abessolo, \& Froi- devaux, 2015).

Estructura Factorial de la Escala de Esperanza Disposicional

La Escala de Esperanza Disposicional para Adultos (Snyder et al., 1991), diseñada para recabar datos sobre la teoría de Snyder, consiste en doce ítems de los cuales ocho están orientados a evaluar ambas dimensiones de la esperanza disposicional y cuatro ítems sirven como distractores. Además, se ha diseñado una Escala de Esperanza-Estado (Snyder et al., 1996) y una Escala de Esperanza para Niños (Snyder et al., 1997). Sin embargo, estas no serán tenidas en cuenta en el presente estudio.

Originalmente, la escala fue validada en estudiantes universitarios de la carrera de psicología y pacientes de dos instituciones en los Estados Unidos (Snyder et al., 1991). Para asegurar la validez de contenido, Snyder y su equipo primeramente escribieron 45 ítems que reflejaran el concepto hipotético de la esperanza. Estos 45 ítems fueron administrados a estudiantes universitarios para luego condensarlos en un instrumento más breve. Los ítems con bajas correlaciones ítem-total fueron descartados, y se mantuvieron 14 ítems con correlaciones ítem-total en niveles aceptables. Luego, Snyder personalmente seleccionó los cuatro ítems que mejor reflejaran el componente de agencia y los cuatro ítems que mejor reflejaran el componente de medios.

Aparte de presentar las evidencias respecto de este estudio, Snyder et al. (1991) recopilaron distintas evidencias de validez y confiabilidad para la misma. El coeficiente alfa de Cronbach para la escala total fue calculado en varias muestras; los resultados oscilaron entre .74 y .84. Para la escala de agencia, los coeficientes alfa fueron de entre .71 y .76 , mientras que para medios fue- 
ron de entre .63 y .80 . Se calculó la confiabilidad test-retest con un intervalo de 3 y 8 semanas $(r=$ .85; $p<.001$; Anderson, 1988, como se citó en Snyder et al., 1991; y $r=.73 ; p<.001$; Harney, 1989, como se citó en Snyder et al., 1991; respectivamente) y de 10 semanas con dos muestras distintas $(r=.76 ; p<.001$ y $r=.82 ; p<.001$; Gibb, 1990, como se citó en Snyder, 1991; y Yoshinobu, 1989, como se citó en Snyder, 1991, respectivamente).

Además, se halló evidencia de validez convergente con escalas de optimismo (correlaciones de .60 y .50 , con valores $p<.005$ en dos estudios), expectativas generalizadas de éxito (correlaciones de .55 y .54 , con valores $p<.005$ en dos estudios), deseabilidad de control $(r=.54 ; p<$ $.005)$, resolución de problemas $(r=-.62 ; p<.005$; puntajes más bajos de esta escala reflejan mayor resolución de problemas), autoestima ( $r=.58 ; p$ $<$.005) y dos escalas de deseabilidad social (correlaciones de .30 y .28; $p<.005)$. Se halló también evidencia de validez divergente con escalas de desesperanza $(r=-.51 ; p<.005)$ y depresión $(r=-.42 ; p<.005$; Gibb, 1990, como se citó en Snyder, 1991; Holleran \& Snyder, 1990, como se citó en Snyder, 1991; Irving, Crenshaw, Snyder, Francis, \& Gentry, 1990, como se citó en Snyder, 1991). Más evidencia de validez concurrente se halló con las subescalas del MMPI y de las Frases Incompletas de Rotter, obteniendo los siguientes resultados: hipocondriasis $(r=-.30 ; p<.001)$, depresión $(r=-.60 ; p<.001)$, histeria $(r=-.35$; $p<.001)$, desviación psicopática $(r=-.43 ; p<$ $.001)$, masculinidad-femineidad ( $r=-.13$; ns), paranoia $(r=-.34 ; p<.001)$, psicastenia $(r=-.52 ; p$ $<.001)$, esquizofrenia $(r=-.46 ; p<.001)$, hipomanía ( $r=-.08$; ns), introversión social $(r=-.59$; $p<.001)$, completamiento de frases $(r=.63 ; p<$ $.001)$. Evidencia de validez discriminante se presentó con respecto a dos subescalas de una escala de autoconciencia ( $r=.06$ y -.03, respectivamente, ns; Gibb, 1990, como se citó en Snyder, 1991).

En cuanto a la validez de constructo, varios estudios confirmatorios experimentales han presentado buena evidencia con respecto al comportamiento de la variable frente a la presencia de estresores, cantidad de objetivos, la dificultad preferida de los objetivos, la evaluación de objetivos vitales, los objetivos académicos, evaluación de consecución de objetivos y la consecución de objetivos (Yoshinobu, 1989; Langelle, 1989; Harris, 1988; Sigmon \& Snyder, 1990; Anderson, 1988; Harney, 1989, todos citados en Snyder et al., 1991). Estos estudios responden a lo hipotetizado sobre la influencia de la esperanza.

Con respecto a la estructura factorial, análisis factoriales favorecieron una estructura de dos factores correlacionados en el cuestionario, en concordancia con los conceptos de agencia y medios (Snyder et al., 1991). Por otro lado, estudios más recientes (Brower, Meijer, Weekers, \& Baneke, 2008) proporcionaron evidencia de que una estructura unidimensional presenta un mejor ajuste para la escala, además de cuestionar la validez incremental que provee la subescala de medios. Por el contrario, Gana, Daigre y Ledrich (2012) apoyaron la estructura de dos factores, y en su estudio, las escalas del cuestionario presentaban una correlación fuerte entre ellas.

Galiana, Oliver, Sancho y Tomás (2014) desarrollaron lo que aparentemente es la primera traducción de la escala al español. Dicho estudio presentó evidencia de análisis factoriales confirmatorios para modelos unifactorial, multidimensional no jerárquico, de segundo orden y de dos factores, hallando mejor ajuste en el caso de la estructura unidimensional. Además, Galiana et al. (2014) señalan que la consistencia interna del cuestionario fue mayor cuando fue tomado como unidimensional. Estos resultados alimentan el de- 
bate sobre la composición de la esperanza.

Al haber resultados contradictorios acerca de la unidimensionalidad vs. multidimensionalidad, es plausible que la Escala de Esperanza se ajuste a un modelo bifactor. Los modelos bifactor evalúan si un conjunto de ítems representa una dimensión general subyacente además de factores específicos (Flores-Kanter, Domínguez-Lara, Trógolo, \& Medrano, 2018). De ser este el caso, la esperanza se conceptualizaría como un constructo unidimensional que incluye factores específicos como agencia y medios.

En Latinoamérica se ha utilizado el instrumento de Snyder para investigación en el contexto de la psicología del deporte (Guillén \& Angulo, 2016), psicología positiva (Bustos, Oliver, \& Galiana, 2015) y la psicología organizacional (Zárate-Torres \& Acosta-Prado, 2015). Para el presente estudio, se elaboró una nueva traducción del instrumento, adaptada a la población paraguaya (ver Tabla 1). Se buscaron traducciones funcionales y no literales, con el fin de que resultaran culturalmente congruentes según las recomendaciones de adaptaciones de cuestionarios de Fernández, Pérez, Alderete, Richaud y Fernández-Liporace (2010).

Buscando aportar nuevos datos a la discusión, nuestra pregunta de investigación indaga si la Escala de Esperanza Disposicional para Adultos en la muestra paraguaya se ajusta a la estructura de dos factores correlacionados de agencia y medios, de dos factores de agencia y medios con esperanza como factor de segundo orden, unifactorial de esperanza, o bifactor con esperanza como factor general y agencia y medios como factores específicos.

\section{Método \\ Participantes}

Se recogieron los datos en universidades públicas y privadas de Paraguay (ver Tabla 1). Un total de 219 estudiantes de grado, mayores de 18 años (55 de sexo masculino, 163 de sexo femenino, uno sin respuesta) y de distintas carreras, completaron los cuestionarios. La edad promedio fue de 23 años de edad (DE = 3.743). Los estudiantes cursaban materias de entre el primer y el séptimo año de sus respectivas carreras. Los participantes fueron informados de que eran libres de completar los cuestionarios, y que no se aplicaría ninguna penalización en caso de no participar. No se ofrecieron incentivos por completar los instrumentos.

La aplicación del instrumento a 164 estu-

Tabla 1

Datos sociodemográficos de la muestra $(\mathrm{N}=219)$.

\begin{tabular}{|c|c|c|}
\hline Característica & $\mathbf{n}$ & $\%$ \\
\hline \multicolumn{3}{|l|}{ Sexo* } \\
\hline Femenino & 163 & 74 \\
\hline Masculino & 55 & 25 \\
\hline \multicolumn{3}{|l|}{ Estado Civil* } \\
\hline Soltero/a & 190 & 87 \\
\hline Viviendo en pareja & 6 & 3 \\
\hline Casado/a & 22 & 10 \\
\hline \multicolumn{3}{|l|}{ Número de hijos* } \\
\hline Ninguno & 190 & 87 \\
\hline Uno & 11 & 5 \\
\hline Dos & 5 & 2 \\
\hline
\end{tabular}

Nota. * Estas variables presentan datos perdidos en el sistema. 
diantes de la muestra se realizó en las aulas durante el horario de clases. Se solicitó autorización a las instituciones y a cada docente para hacer uso de los últimos 30 minutos de clase para este fin. Al terminar, los participantes depositaron sus cuestionarios completados en una mesa designada antes de retirarse del aula. Por otro lado, 55 estudiantes completaron una versión de los cuestionarios diseñada en Qualtrics. Se alcanzó a dichos estudiantes por medio de un muestreo tipo bola de nieve compartiendo los cuestionarios en redes sociales como Facebook y Twitter. Antes del levantamiento de los datos, todos los participantes leyeron y firmaron formularios de consentimiento informado. El procedimiento de este estudio fue aprobado previamente por el comité de ética de la Universidad de Kansas, afiliación institucional de la primera autora en aquel momento.

Análisis de poder y tamaño muestral. Varios análisis de poder fueron realizados utilizando la calculadora de Preacher y Coffman (2006) para asegurar un tamaño muestral adecuado para este estudio. Los cálculos de grados de libertad tuvieron en cuenta los modelos hipotéticos a contrastar. Para comprobar el poder para evaluar el ajuste total del modelo, se realizó el análisis de ajuste pobre de MacCallum, Browne y Sugawara (1996). El análisis de ajuste pobre nos permite saber si, dado el tamaño muestral, se podría rechazar una hipótesis de ajuste pobre en la muestra si el ajuste de la población fuera bueno y viceversa. Basado en los modelos, los grados de libertad van entre 12 y 20. Se especificó una hipótesis nula de pobre ajuste con un valor de RMSEA de .10 o mayor, $\mathrm{y}$ una hipótesis alternativa de buen ajuste con un valor de RMSEA de .02 o menor. Para 12 grados de libertad, .80 de poder, y .05 de valor alfa, el análisis de poder indicó que el tamaño muestral mínimo sería de $\mathrm{N}=166$; para 20 grados de libertad, .80 de poder, y .05 de alfa, el tamaño muestral mínimo sería de $\mathrm{N}=119$. Por lo tanto, el tamaño muestral obtenido de 219 tiene suficiente poder para los análisis realizados.

\section{Instrumentos}

Cuestionario sociodemográfico. Incluyó preguntas relacionadas con la edad, sexo, nombre de la universidad, carrera, años en la carrera, si era el primer miembro de su familia en asistir a la universidad, grado de seguridad en la elección de la carrera, ocupación de los padres, nivel de instrucción, estado civil, número de hijos, y quién había elegido la carrera que se encontraban estudiando (ej., si la habían elegido ellos o sus padres). Este cuestionario fue generado por la primera autora en colaboración con otros investigadores para estudios en la misma línea.

\section{Escala de Esperanza Disposicional para Adul-} tos (Snyder et al., 1991). Consta de 12 reactivos dispuestos en dos subescalas: cuatro para la subescala de Agencia, cuatro para la de Medios y cuatro ítems adicionales que actúan como distractores. Las opciones de respuesta se presentan en una escala Likert de ocho puntos, que va desde 1 (Definitivamente falso) hasta 8 (Definitivamente verdadero). Los puntajes en las subescalas son aditivos y forman un puntaje total. El coeficiente alfa de Cronbach para la presente muestra es de .78 para la subescala de Agencia, de .69 para la subescala de Medios y de .83 para la escala total. Como el instrumento original de Snyder et al. (1991) fue redactado en inglés, para el presente estudio utilizamos el procedimiento de traducción y re-traducción para adaptarlo a la población hispanoparlante en Paraguay, siguiendo los estándares de la comunidad científica enfatizados en Fernández et al. (2010). Un experto en español e inglés con conocimientos de psicología tradujo la 
versión original de los instrumentos al español. Posteriormente, otro experto en ambos idiomas tradujo la versión en español de nuevo al inglés. Un tercer experto en ambos idiomas comparó ambas versiones para asegurarse de que los reactivos fueran análogos y mantuvieran su significado.

\section{Plan de Análisis de Datos}

Primero, se realizaron análisis descriptivos de los ítems y las subescalas que componen la Escala de Esperanza. Se midió asimetría y curtosis tomando como indicadores de normalidad su transformación a puntajes $z$ dividiendo los puntajes por su error estándar y verificando su significancia.

Se realizaron análisis factoriales confir-

Tabla 2

Reactivos de la Escala de Esperanza Traducidos al Español.

\begin{tabular}{cl}
$\begin{array}{c}\text { Número de } \\
\text { reactivo }\end{array}$ & \multicolumn{1}{c}{ Enunciado del reactivo } \\
\hline 1 & Puedo pensar en muchas maneras de salir de un embrollo. \\
2 & Persigo mis metas con energía. \\
3 & Me siento cansado/a la mayoría del tiempo. \\
4 & Hay muchas maneras de solucionar cualquier problema. \\
5 & Me siento rápidamente vencido/a en una discusión. \\
6 & Puedo pensar en muchas maneras de conseguir las cosas que son importantes para mi vida. \\
7 & Me preocupo por mi salud. \\
8 & Incluso cuando otros se sienten desanimados, yo sé que puedo encontrar una manera de solucionar el \\
9 & problema. \\
10 & Mis experiencias pasadas me han preparado bien para mi futuro. \\
11 & Usualmente me encuentro preocupado/a por algo. \\
12 & Cumplo las metas que me trazo. \\
\hline
\end{tabular}

matorios en el paquete lavaan del programa $R$ (Rosseel, 2012) mediante estimación de máxima probabilidad; además, se utilizó la estimación robusta de máxima probabilidad con la corrección de chi-cuadrado Satorra-Bentler, al haber desviación de la normalidad en la distribución de los puntajes según recomendación de Finney y DiStefano (2013), quienes además sugieren la presentación de los resultados con ambos métodos. Estos análisis compararon cuatro modelos: la esperanza como un constructo de dos factores correlacionados, la esperanza como un constructo de dos factores con un factor de segundo orden, la esperanza como un constructo unifactorial y la esperanza como un constructo bifactor.

Los modelos en el análisis factorial confirmatorio también se llaman modelos de medición, y definen a los constructos latentes mediante múltiples indicadores corrigiendo así el error de medición (Little, 2013). De este modo, pueden establecer la evidencia de validez basada en el contenido de estos indicadores separando a los confiables de los no confiables. Los parámetros poblacionales en modelos de ecuaciones estructurales como los análisis factoriales confirmatorios no incluyen sesgo y por ende son más exactos y más generalizables (Little, 2013).

La varianza factorial se fijó mediante el método de configuración de la escala (scale setting; Little, 2013). Las estadísticas de bondad de ajuste siguieron las sugerencias de $\mathrm{Hu}$ y Bentler (1999) y Little (2013) acerca de un ajuste aceptable con CFI $>.90$, RMSEA $<.08$ y $\mathrm{SRMR}<.11$, o ajuste excelente con $\mathrm{CFI}>.95, \mathrm{RMSEA}<.05$ y $\mathrm{SRMR}$ 
$<$.06. Para comparar los modelos, se tomaron en cuenta las convenciones para los índices de ajuste; en caso de ser apropiadas, se utilizó la regla de Cheung y Rensvold (2002) de continuar evaluando el siguiente modelo si el cambio en CFI fuera menor $\mathrm{a}<.01$.

Los datos perdidos en las variables de interés estuvieron entre 0 y $1.4 \%$ (.004\% de los puntos totales) y fueron considerados falta completamente al azar o MCAR (missing completely at random; Little, 2013). Fueron estimados en los análisis de máxima probabilidad mediante el uso del método de información completa (full estimation maximum likelihood o FIML en lavaan). Los análisis con la corrección robusta de Satorra-Bentler no admiten el uso de la información completa, y en ese caso la muestra final para estos análisis fue de 212 participantes debido a la eliminación de los casos con datos perdidos.

Además, se evaluaron índices complementarios para estimar la adecuación del modelo bifactor, como la varianza total explicada (ECV), la varianza total explicada por ítem (IECV), el índice de replicabilidad del constructo (H-index), el porcentaje de correlaciones no contaminadas (PUC) y los coeficientes de confiabilidad Omega. Para el cálculo de dichos índices, se utilizaron las calculadoras disponibles en línea de Dueber (2017) y Hammer (2016). Estos índices son especialmente importantes para una interpretación apropiada, ya que los modelos bifactor tienden a presentar mejor ajuste que los análisis factoriales confirmatorios solamente debido a la manera en la que se los especifica (Flores-Kanter et al., 2018).

\section{Resultados}

Los puntajes medios en cada ítem (ver Tabla 3) oscilan entre los tres y los siete puntos. Los desvíos estándar se encuentran entre uno y dos puntos para cada caso.

Los valores de asimetría y curtosis observados en la Tabla 3, transformados a puntajes $z$, oscilan entre -5.95 y -4.61 para asimetría y 1.15 y 4.17 para curtosis, dándonos asimetría y curtosis significativas $(p<.001)$. Por lo tanto, se presentan tanto los análisis con estimación de máxima

Tabla 3

Indicadores Descriptivos.

\begin{tabular}{|c|c|c|c|c|c|}
\hline Ítems & $\mathbf{M}$ & DE & Asimetría & Curtosis & $\alpha$ de Cronbach \\
\hline $1-$ Medios & 6.48 & 1.22 & -.89 & .86 & \\
\hline 2 - Agencia & 6.49 & 1.23 & -.79 & .53 & \\
\hline 3 - Distractor & 5.00 & 1.87 & -.36 & -.88 & \\
\hline 4 - Medios & 6.81 & 1.28 & -1.50 & 2.89 & \\
\hline 5 - Distractor & 3.38 & 2.04 & .58 & -.79 & \\
\hline $6-$ Medios & 6.70 & 1.12 & -.79 & .09 & \\
\hline 7 - Distractor & 6.35 & 1.60 & -1.11 & .91 & \\
\hline 8 - Medios & 6.43 & 1.33 & -1.24 & 2.22 & \\
\hline 9 -Agencia & 6.60 & 1.41 & -1.31 & 2.09 & \\
\hline 10 - Agencia & 5.96 & 1.41 & -.89 & .71 & \\
\hline 11 - Distractor & 5.86 & 1.56 & -.80 & .41 & \\
\hline 12 - Agencia & 6.30 & 1.26 & -.74 & .39 & \\
\hline Escala de Agencia & 25.35 & 4.13 & -.99 & 1.38 & .69 \\
\hline Escala de Medios & 26.48 & 3.54 & -.76 & .38 & .78 \\
\hline Total & 51.89 & 6.91 & -.90 & 1.09 & .83 \\
\hline
\end{tabular}


Tabla 4

Matriz de Covarianzas y Correlaciones entre Reactivos.

\begin{tabular}{ccccccccc}
\hline & \multicolumn{3}{c}{ Medios } & \multicolumn{5}{c}{ Agencia } \\
\hline Reactivos & $\mathbf{1}$ & $\mathbf{4}$ & $\mathbf{6}$ & $\mathbf{8}$ & $\mathbf{2}$ & $\mathbf{9}$ & $\mathbf{1 0}$ & $\mathbf{1 2}$ \\
\hline 1 & 1.445 & .432 & .565 & .688 & .521 & .560 & .458 & .454 \\
4 & .281 & 1.642 & .311 & .582 & .453 & .536 & .688 & .465 \\
6 & .419 & .216 & 1.256 & .641 & .559 & .360 & .477 & .437 \\
8 & .438 & .347 & .437 & 1.709 & .749 & .808 & .736 & .702 \\
2 & .351 & .285 & .403 & .463 & 1.531 & .751 & .799 & .848 \\
9 & .327 & .294 & .225 & .434 & .426 & 2.033 & .953 & .842 \\
10 & .268 & .378 & .300 & .397 & .455 & .471 & 2.014 & .884 \\
12 & .302 & .290 & .311 & .429 & .548 & .472 & .498 & 1.567
\end{tabular}

Nota. Las covarianzas se presentan en la diagonal y por encima de la diagonal. Las correlaciones se presentan debajo de la diagonal, en cursiva y negrita.

probabilidad, así como los análisis factoriales confirmatorios con chi-cuadrado de SatorraBentler que tienen en cuenta la desviación de la normalidad. En la Tabla 4 puede apreciarse la matriz de correlaciones y covarianzas, encontrándose correlaciones entre los reactivos que componen al instrumento.

Los datos (Tabla 5) indican la bondad de ajuste de los modelos con análisis de máxima probabilidad y chi-cuadrado de Satorra-Bentler, que presentan resultados similares. Según estos análisis, tanto la estructura de dos factores correlacionados, la estructura de dos factores con un factor de segundo orden, la unifactorial y la bifactor se ajustan de manera excelente, siguiendo los criterios de interpretación de ajuste propuestos por $\mathrm{Hu}$ y Bentler (1999) y Little (2013).

El CFI del modelo de dos factores correlacionados, del modelo de dos factores con un factor de segundo orden y del modelo bifactor fueron idénticos en los análisis de Satorra-Bentler, y prácticamente idénticos en los análisis con estimación de máxima probabilidad. No se pudo calcular índices de ajuste para el modelo bifactor con estimación de máxima probabilidad por fallas de convergencia luego de 10000 iteraciones. En comparación con el modelo unifactorial, el cambio del CFI fue mayor a .01 para ambas estimaciones, lo que indica que los modelos unifactoriales tienen un peor ajuste (Cheung \& Rensvold, 2002).

Al presentar excelente ajuste, los modelos de dos factores correlacionados, dos factores con un factor de segundo orden y bifactor, fue necesario recurrir a los índices complementarios para poder elucidar la unidimensionalidad o bidimensionalidad de la Escala de Esperanza Disposicional.

La varianza común explicada total del modelo bifactor se computó con la calculadora en línea de Dueber (2017) y fue de ECV = .768 para el factor general, ECV-S $=.273$ para el factor específico de agencia y ECV-S = .177 para el factor específico de medios. El porcentaje de correlaciones no contaminadas o PUC fue de .571, que indica las covarianzas o correlaciones que solamente reflejan variación de la dimensión general y no están contaminadas con multidimensionalidad (Flores-Kanter et al., 2018).

Además, se calculó la varianza común explicada del ítem o IECV, que indica si la varianza en las respuestas a un ítem se puede atribuir a la 
Tabla 5

Índices de Bondad de Ajuste de los Análisis Factoriales Confirmatorios.

\begin{tabular}{|c|c|c|c|c|c|c|c|c|c|c|}
\hline & Modelo & $d f$ & $\chi^{2}$ & $p \chi^{2}$ & CFI & AIC & RMSEA & SRMR & $\begin{array}{c}\text { Modelo de } \\
\text { Comparación }\end{array}$ & $\Delta \mathrm{CFI}$ \\
\hline 1.1 & $\begin{array}{l}\text { Dos factores correlaciona- } \\
\text { dos, estimación de máxima } \\
\text { probabilidad }(N=219)\end{array}$ & 19 & 23.853 & .202 & .989 & 5393.119 & .034 & .033 & & \\
\hline 1.2 & $\begin{array}{c}\text { Dos factores correlaciona- } \\
\text { dos, Satorra-Bentler } \\
(N=212)\end{array}$ & 19 & 15.475 & .692 & 1.000 & 5219.461 & .000 & .034 & & \\
\hline 2.1 & $\begin{array}{l}\text { Dos factores con un factor de } \\
\text { segundo orden, estimación } \\
\text { de máxima probabilidad }\end{array}$ & 18 & 23.853 & .160 & .987 & 5395.119 & .039 & .033 & 2.1 vs 1.1 & .002 \\
\hline 2.2 & $\begin{array}{l}(N=219) \\
\text { Dos factores con un factor } \\
\text { de segundo orden, Sato- } \\
\text { rra-Bentler }(N=212)\end{array}$ & 18 & 15.599 & .621 & 1.000 & 5221.461 & .000 & .033 & 2.2 vs 1.2 & .000 \\
\hline 3.1 & $\begin{array}{l}\text { Unifactorial, estimación de } \\
\text { máxima probabilidad } \\
(N=219)\end{array}$ & 20 & 40.731 & .004 & .954 & 5407.997 & .069 & .044 & 3.1 vs 1.1 & .035 \\
\hline 3.2 & $\begin{array}{l}\text { Unifactorial, Satorra-Bentler } \\
\qquad(N=212)\end{array}$ & 20 & 28.304 & .102 & .968 & 5235.857 & .044 & .044 & 3.2 vs 1.2 & .032 \\
\hline 4.1 & $\begin{array}{l}\text { Bifactor, estimación de máxi- } \\
\text { ma probabilidad }(N=219)^{*}\end{array}$ & - & - & - & - & - & - & - & - & - \\
\hline 4.2 & $\begin{array}{l}\text { Bifactor, Satorra-Bentler } \\
\qquad(N=212)\end{array}$ & 12 & 10.752 & .550 & 1.000 & 5209.093 & .000 & .027 & 4.2 vs 1.2 & .000 \\
\hline
\end{tabular}

Nota. No fue posible calcular índices de ajuste para el modelo 4.1, bifactor con estimación de máxima probabilidad, debido a que dicho modelo no logró convergencia luego de 10000 iteraciones y por ende no es confiable.

varianza en la dimensión general; puede ser útil para evaluar la unidimensionalidad e inclusive extraer un conjunto de ítems que reflejen el contenido de la dimensión general (Stucky \& Edelen, 2015). La IECV en la Escala de Esperanza estuvo entre .534 y .965 , con cinco ítems por encima del valor de .80 que sugiere que dicho ítem refleja la dimensión general (ver Tabla 6).

Se calculó el índice de replicabilidad del constructo o H-index (Rodríguez, Reise, \& Haviland, 2016) utilizando la calculadora de Hammer (2016), y se obtuvo un índice de .942 para el factor general de esperanza, .578 para el factor específico de agencia y .371 para el factor específico de medios; para el modelo de dos factores correlacionados, el H-index fue de .96 tanto para la subescala de Agencia como para la de Medios. Valores por encima de .80 indican que la variable latente está bien definida y el constructo podrá ser replicado en estudios posteriores (Rodríguez et al., 2016).

La confiabilidad fue estudiada con los coeficientes Omega (Flores-Kanter et al., 2018). 
Tabla 6

Carga Factorial e IECV de la Escala de Esperanza en el Modelo Bifactor.

\begin{tabular}{ccccc}
\hline Ítems & Factor General & $\begin{array}{c}\text { Factor Específico } \\
\text { Medios }\end{array}$ & $\begin{array}{c}\text { Factor Específico } \\
\text { Agencia }\end{array}$ & IECV \\
\hline 1 - Medios & .657 & .326 & & .802 \\
4 - Medios & .662 & -.124 & & .966 \\
6 - Medios & .583 & .545 & & .534 \\
8 - Medios & .938 & .179 & & .965 \\
2 - Agencia & .782 & & .386 & .804 \\
9 - Agencia & .817 & & .393 & .812 \\
10 - Agencia & .830 & & .445 & .777 \\
12 - Agencia & .726 & .659 & .548 \\
\hline
\end{tabular}

El coeficiente Omega $(\omega)$ es un estimativo de la confiabilidad interna del constructo multidimensional, basado en el modelo especificado, en donde para el factor general se consideran todos los ítems, y en este estudio fue de .952; para el Omega de subescalas $\left(\omega_{\mathrm{s}}\right)$ se consideran solamente los ítems que cargan en ese factor específico, hallándose $\omega_{\mathrm{s}}=.960$ para la subescala de Agencia y $\omega_{\mathrm{s}}$ $=.859$ para la subescala de Medios. El coeficiente Omega jerárquico u OmegaH $\left(\omega_{\mathrm{h}}\right)$ indica la proporción de la varianza que puede ser atribuida a diferencias individuales en el factor general; en este estudio, $\omega_{\mathrm{h}}=.848$. El coeficiente Omega jerárquico de subescalas u OmegaHS $\left(\omega_{\mathrm{hs}}\right)$ indica la proporción de la varianza correspondiente a una subescala luego de controlar la varianza atribuida al factor general, y se halló $\omega_{\mathrm{hs}}=.252$ para la subescala de Agencia y $\omega_{\mathrm{hs}}=.083$ para la subescala de Medios.

\section{Discusión}

El presente estudio se centró en la validación factorial de la Escala de Esperanza Disposicional en Adultos (Snyder et al., 1991). Se han propuesto distintos modelos de ajuste para dicho instrumento, desde el unifactorial (Brower et al., 2008; Galiana et al., 2014) hasta el de dos factores (Snyder et al., 1991; Gana et al., 2012). En este trabajo, se aplicó un análisis factorial confir- matorio para probar el ajuste con un modelo unifactorial, un modelo de dos factores correlacionados, un modelo de dos factores con un factor de segundo orden y un modelo bifactor.

Los datos muestran que el instrumento presenta ajuste óptimo con los tres modelos. Sin embargo, a nuestro criterio, el modelo con mejor ajuste es el bifactor. Las subescalas de Agencia y Medios presentan puntajes más bajos en el alfa de Cronbach (.78 en la subescala de Agencia, 69 en la subescala de Medios) en comparación con la escala total con un alfa de Cronbach de .83. El ajuste unifactorial también técnicamente presenta un ajuste excelente en esta muestra, aunque ligeramente inferior a los modelos de dos factores y bifactor.

Como sugieren Reise, Scheines, Widaman, y Haviland (2013), cuando los valores de PUC son inferiores a .80 (en este estudio, $\mathrm{PUC}=.571)$, la ECV general mayor a .60 (en este estudio, ECV $=.768)$ y OmegaH del factor general mayor a .70 (en este estudio, $\omega_{\mathrm{h}}=.848$ ) cierta multidimensionalidad existe, pero no es lo suficientemente severa para descalificar la interpretación de la escala como principalmente unidimensional. Por ende, según estos resultados, la esperanza es un constructo preponderantemente unidimensional con ciertas características específicas.

Estos resultados apoyan la estructura teórica que se ha propuesto para la esperanza (Snyder et 
al., 1991; Gana et al., 2012) como un constructo general incluyendo dos factores específicos, y explican por qué otros estudios pudieron hallar apoyo para una estructura unidimensional (Galiana et al., 2014). Al ajustarse la Escala de Esperanza Disposicional a un modelo bifactor, se resalta la unidimensionalidad primaria del constructo de esperanza, a pesar de la presencia de cierta multidimensionalidad; esto implica que estas escalas no son independientes, sino que ambas son parte específica de la esperanza general. A nivel teórico, esto podría implicar constante interacción y retroalimentación entre ambos componentes específicos de la esperanza como estado general.

Inclusive, basándonos en los IECV, se podría crear una versión breve de la Escala de Esperanza Disposicional que refleje el constructo de la esperanza como unidimensional (Stucky \& Edelen, 2015); esta versión se presenta en la Tabla 7.

Los resultados también indican que la traducción de la escala presenta validez factorial y consistencia interna, lo que favorece su utilización en investigación y práctica con muestras paraguayas y potencialmente de toda Latinoamérica. A partir de estas conclusiones, la medición de la esperanza con el presente instrumento podrá ser incluida en modelos como factor o como mediador de otros factores. En resumen, los resultados indican que la escala es sumamente útil para la medición de la esperanza disposicional.
Como limitaciones del estudio, se destaca que los participantes fueron encontrados en ámbitos universitarios, lo que quizás limitaría las conclusiones a estratos sociales con un nivel académico avanzado. Al ser un muestreo por conveniencia y bola de nieve, podría no ser generalizable a la población universitaria general. Además, gran parte de la población paraguaya habla solamente idioma guaraní, teniendo conocimientos no siempre óptimos del español. Por ese motivo, la escala no podría ser aplicada en esos estratos.

\section{Conclusión}

El análisis factorial confirmatorio realizado en este estudio permite concluir que la Escala de Esperanza Disposicional en Adultos (Snyder et al., 1991) se ajusta de mejor manera a un modelo bifactor; por lo tanto, la esperanza es un factor general que engloba agencia y medios como factores específicos. La traducción de esta escala al español presenta validez factorial y consistencia interna para muestras paraguayas, lo que permite su utilización específicamente en individuos con buen manejo del idioma español. Replicaciones de la estructura factorial en otras poblaciones latinoamericanas servirán para generalizar la utilidad del instrumento. Al tener un instrumento que presenta evidencias de validez, se puede conti-

\section{Tabla 7}

Reactivos de la Escala de Esperanza para Versión Breve.

\begin{tabular}{cl}
\hline $\begin{array}{c}\text { Número original } \\
\text { de reactivo }\end{array}$ & \multicolumn{1}{c}{ Enunciado del reactivo } \\
\hline 1 & Puedo pensar en muchas maneras de salir de un embrollo. \\
2 & Persigo mis metas con energía. \\
4 & $\begin{array}{l}\text { Hay muchas maneras de solucionar cualquier problema. } \\
\text { Incluso cuando otros se sienten desanimados, yo sé que puedo encontrar una manera de solucionar el } \\
\text { problema. } \\
9\end{array}$ \\
\hline
\end{tabular}


nuar evaluando la relación entre características de personalidad o rasgos conductuales que pudieran estar relacionados con la esperanza, o moderados o mediados por esta.

\section{Referencias}

Brower, D., Meijer, R. R., Weekers, A. M., \& Baneke, J. J. (2008). On the dimensionality of the Dispositional Hope Scale. Psychological Assessment, 20(3), 310315. doi: 10.1037/1040-3590.20.3.310

Bustos, V., Oliver, A., \& Galiana, L. (2015). Validación del autoconcepto forma 5 en universitarios peruanos: Una herramienta para la psicología positiva. Psicologia: Reflexão e Crítica, 28(4), 690-697. doi: 10.1590/1678-7153.201528406

Cheung, G. W., \& Rensvold, R. B. (2002). Evaluating goodness-of-fit indexes for testing measurement invariance. Structural Equation Modeling, 9(2), 233255. doi: 10.1207/s15328007sem0902_5

Dueber, D. M. (2017). Bifactor Indices Calculator: A Microsoft Excel-based tool to calculate various indices relevant to bifactor CFA models [software de cálculo]. Disponible en https://doi.org/10.13023/edp. tool.01, https://uknowledge.uky.edu/edp_tools/1/

Fernández, A., Pérez, E., Alderete, A. M., Richaud, M. C., \& Fernández-Liporace, M. (2010). ¿Construir o adaptar tests psicológicos? Diferentes respuestas a una cuestión controvertida. Revista Evaluar, 10(1), 60-74. Recuperado de https://revistas.unc.edu.ar/index.php/revaluar

Flores-Kanter, P. E., Dominguez-Lara, S., Trógolo, M. A., $\&$ Medrano, L. A. (2018). Best practices in the use of bifactor models: Conceptual grounds, fit indices and complementary indicators. Revista Evaluar, 18(3), 44-48. Recuperado de https://revistas.unc.edu.ar/index.php/revaluar
Finney, S. J., \& DiStefano, C. (2013). Nonnormal and categorical data in structural equation modeling. En G. R. Hancock \& R. O. Mueller (Eds.), Structural equation modeling: A second course ( $2^{\mathrm{a}}$ ed., pp. 439-492). Charlotte, NC: Information Age.

Galiana, L., Oliver, A., Sancho, P., \& Tomás, J. M. (2014). Dimensionality and validation of the Dispositional Hope Scale in a Spanish sample. Social Indicators Research, 120(1), 297-308. doi: 10.1007/s11205014-0582-1

Gana, K., Daigre, S., \& Ledrich, J. (2012). Psychometric properties of the French version of the Adult Dispositional Hope Scale. Assessment, 20(1), 114-118. doi: $10.1177 / 1073191112468315$

Guillén, F., \& Angulo, J. (2016). Análisis de rasgos de personalidad positiva y bienestar psicológico en personas mayores practicantes de ejercicio físico vs no practicantes. Revista Iberoamericana de Psicología del Ejercicio y el Deporte, 11(1), 113-122. Recuperado de http://www.riped-online.com/index.php/riped/ inicio

Hammer, J. H. (2016). Construct Replicability Calculator: A Microsoft Excel-based tool to calculate the Hancock and Mueller (2001) H index [software de cálculo]. Recuperado de http://drjosephhammer.com el 06/03/2019

Hirschi, A., Abessolo, M., \& Froidevaux, A. (2015). Hope as a resource for career exploration: Examining incremental and cross-lagged effects. Journal of Vocational Behavior, 86, 38-47. doi: 10.1016/j. jvb.2014.10.006

Hu, L., \& Bentler, P. M. (1999). Cutoff criteria for fit indexes in covariance structure analysis: Conventional criteria versus new alternatives. Structural Equation Modeling: A Multidisciplinary Journal, 6(1), 1-55. doi: 10.1080/10705519909540118

Little, T. D. (2013). Longitudinal Structural Equation Mo- 
deling. New York, NY: Guilford.

MacCallum, R. C., Browne, M. W., \& Sugawara, H. M. (1996). Power analysis and determination of sample size for covariance structure modeling. Psychological Methods, 1(2), 130-149. doi: 10.1037/1082989X.1.2.130

Preacher, K. J., \& Coffman, D. L. (2006). Computing power and minimum sample size for RMSEA [software de cálculo]. Recuperado de http://quantpsy.org

Reise, S. P., Scheines, R., Widaman, K. F., \& Haviland, M. G. (2013). Multidimensionality and structural coefficient bias in structural equation modeling: A bifactor perspective. Educational and Psychological Measurement, 73(1), 5-26. Recuperado de https://psycnet. apa.org/record/2012-34046-001

Rodriguez, A., Reise, S. P., \& Haviland, M. G. (2016). Applying bifactor statistical indices in the evaluation of psychological measures. Journal of Personality Assessment, 98(3), 223-237. doi: 10.1080/00223891.2015.1089249

Rosseel, Y. (2012). lavaan: An R package for structural equation modeling. Journal of Statistical Software, 48(2), 1-36. doi: 10.18637/jss.v048.i02

Snyder, C. R. (2002). Hope theory: Rainbows in the mind. Psychological Inquiry: An International Journal for the Advancement of Psychological Theory, 13(4), 249-275. doi: 10.1207/S15327965PLI1304 01

Snyder, C. R., Feldman, D. B., Taylor, J. D., Schroeder, L. L., \& Adams, V. H. (2000). The roles of hopeful thinking in preventing problems and enhancing strengths. Applied and Preventive Psychology, 9(4), 249269. doi: 10.1016/S0962-1849(00)80003-7

Snyder, C. R., Harris, C., Anderson, J. R., Holleran, S. A., Irving, L. M., Sigmon, S. T. ... Harney, P. (1991). The will and the ways: Development and validation of an individual-differences measure of hope. Journal of Personality and Social Psychology, 60(4),
570-585. doi: 10.1037/0022-3514.60.4.570

Snyder, C. R., Hoza, B., Pelham, W. E., Rapoff, M., Ware, L., Danovsky, M., ... Stahl, K. J. (1997). The development and validation of the Children's Hope Scale. Journal of Pediatric Psychology, 22(3), 399-421. doi: 10.1093/jpepsy/22.3.399

Snyder, C. R., Sympson, S. C., Ybasco, F. C., Borders, T. F., Babyak, M. A., \& Higgins, R. L. (1996). Development and validation of the State Hope Scale. Journal of Personality and Social Psychology, 70(2), 321335. doi: 10.1037/0022-3514.70.2.321

Stucky, B. D., \& Edelen, M. O. (2015). Using hierarchical IRT models to create unidimensional measures from multidimensional data. En S. P. Reise \& D. A. Revicki (Eds.), Handbook of item response theory modeling: Applications to typical performance assessment (pp. 183-206). New York, NY: Routledge.

Yarcheski, A., \& Mahon, N. E. (2014). Meta-analyses of predictors of hope in adolescents. Western Journal of Nursing Research, 38(3), 345-368. doi: $10.1177 / 0193945914559545$

Zárate-Torres, R. A., \& Acosta-Prado, J. C. (2015). Esperanza y atributos de los colaboradores. Suma de Negocios, 6(14), 178-182. doi: 10.1016/j.sumneg.2015.07.002 